\title{
The physical structure of the point-symmetric and quadrupolar planetary nebula NGC 6309
}

\author{
R. Vázquez ${ }^{1}$, L. F. Miranda ${ }^{2}$, L. Olguín ${ }^{1,3}$, S. Ayala ${ }^{3,4}$, J. M. Torrelles ${ }^{5}$, M. E. Contreras ${ }^{1}$, and P. F. Guillén ${ }^{1}$ \\ ${ }^{1}$ Instituto de Astronomía, Universidad Nacional Autónoma de México, Apdo. Postal 877, 22800 Ensenada, B. C., Mexico \\ e-mail: [vazquez;mcontreras; fguillen]@astrosen.unam.mx \\ 2 Instituto de Astrofísica de Andalucía, CSIC, PO Box 3004, 18080 Granada, Spain \\ e-mail: lfm@iaa.es \\ 3 Instituto de Astronomía, Universidad Nacional Autónoma de México, Apdo. Postal 70-264, 04510 México, D. F., Mexico \\ e-mail: lorenzo@astrosen.unam.mx \\ ${ }^{4}$ Centro de Radioastronomía y Astrofísica, Universidad Nacional Autónoma de México, Apdo. Postal 3-72 (Xangari), 58089 Morelia, \\ Mich., Mexico \\ e-mail: s.ayala@astrosmo.unam.mx \\ 5 Instituto de Ciencias del Espacio (CSIC) - IEEC, Facultat de Física, Universitat de Barcelona, Av. Diagonal 647, 08028 Barcelona, \\ Spain \\ e-mail: torrelles@ieec.fcr.es
}

Received 21 August 2007 / Accepted 18 December 2007

\begin{abstract}
Aims. We analyse the point-symmetric planetary nebula NGC 6309 in terms of its three-dimensional structure and of internal variations of the physical conditions to deduce the physical processes involved in its formation.

Methods. We used VLA-D 13.6-cm continuum, ground-based, and HST-archive imaging as well as long slit high- and low-dispersion spectroscopy.

Results. The low-dispersion spectra indicate a high excitation nebula, with low to medium variations of its internal physical conditions $\left(10600 \mathrm{~K} \lesssim T_{\mathrm{e}}[\mathrm{O} \mathrm{III}] \lesssim 10900 \mathrm{~K} ; 10100 \mathrm{~K} \lesssim T_{\mathrm{e}}[\mathrm{N} \mathrm{III}] \lesssim 11800 \mathrm{~K} ; 1440 \mathrm{~cm}^{-3} \lesssim N_{\mathrm{e}}[\mathrm{S} \mathrm{III}] \lesssim 4000 \mathrm{~cm}^{-3} ; 1700 \mathrm{~cm}^{-3} \lesssim N_{\mathrm{e}}[\mathrm{Cl}\right.$ III $] \lesssim$ $2600 \mathrm{~cm}^{-3} ; 1000 \mathrm{~cm}^{-3} \lesssim N_{\mathrm{e}}\left[\right.$ Ar IV] $\lesssim 1700 \mathrm{~cm}^{-3}$ ). The radio continuum emission indicates a mean electron density of $\simeq 1900 \mathrm{~cm}^{-3}$, emission measure of $5.1 \times 10^{5} \mathrm{pc} \mathrm{cm}^{-6}$, and an ionised mass $M(\mathrm{H} \mathrm{II}) \simeq 0.07 M_{\odot}$. In the optical images, the point-symmetric knots show a lack of [N II] emission as compared with similar features previously known in other PNe. A rich internal structure of the central region is seen in the HST images, resembling a deformed torus. Long slit high-dispersion spectra reveal a complex kinematics in the central region, with internal expansion velocities ranging from $\simeq 20$ to $30 \mathrm{~km} \mathrm{~s}^{-1}$. In addition, the spectral line profiles from the external regions of NGC 6309 indicate expanding lobes $\left(\simeq 40 \mathrm{~km} \mathrm{~s}^{-1}\right)$ as those generally found in bipolar nebulae. Finally, we have found evidence for the presence of a faint halo, possibly related to the envelope of the AGB-star progenitor.

Conclusions. Our data indicate that NGC 6309 is a quadrupolar nebula with two pairs of bipolar lobes whose axes are oriented PA = $40^{\circ}$ and $\mathrm{PA}=76^{\circ}$. Equatorial and polar velocities for these two pairs of lobes are 29 and $86 \mathrm{~km} \mathrm{~s}^{-1}$ for the bipolar system at PA $=$ $40^{\circ}$ and 25 and $75 \mathrm{~km} \mathrm{~s}^{-1}$ for the bipolar system at $\mathrm{PA}=76^{\circ}$. There is also a central torus that is expanding at $25 \mathrm{~km} \mathrm{~s}^{-1}$. Kinematical age for all these structures is around 3700 to $4000 \mathrm{yr}$. We conclude that NGC 6309 was formed by a set of well-collimated bipolar outflows (jets), which were ejected in the initial stages of its formation as a planetary nebula. These jets carved the bipolar lobes in the previous AGB wind and their remnants are now observed as the point-symmetric knots tracing the edges of the lobes.
\end{abstract}

Key words. planetary nebulae: individual: NGC 6309 - ISM: kinematics and dynamics - ISM: abundances

\section{Introduction}

In spite of all the work that up to now has been done to understand the origin of planetary nebulae (PNe) morphologies and their evolution (e.g., Kwok et al. 1978; Kahn \& West 1985; Balick 1987; Icke 1988; Mellema 1995; Perinotto et al. 2004; Rijkhorst et al. 2005; Schönberner et al. 2005, 2007), some morphological structures still remain as unsolved problems. In particular, the so-called point-symmetry (Stanghellini et al. 1993; Gonçalves et al. 2003) appears enigmatic. Some observational studies about point-symmetric PNe (e.g. Miranda \& Solf 1992; López et al. 1993) relate the formation of these objects to collimated outflows from a precessing central source known as bipolar rotating episodic jets (BRETs, see López et al. 1995). However, in some cases, there are not definite proofs about the jet nature of these features as they are not confirmed by spectroscopic studies (e.g. Vázquez et al. 1999a, 2002). Although some theoretical models have intended to explain point-symmetric PNe (e.g., Cliffe et al. 1995; Livio \& Pringle 1996, 1997; García-Segura \& López 2000; Rijkhorst et al. 2004), the origin and shaping of this kind of objects remains a puzzle.

NGC 6309 is a PN whose morphology strongly suggests a BRET scenario for its origin, as can be seen in the $\mathrm{H} \alpha+[\mathrm{N} \mathrm{II}]$ and [O III] images by Schwarz et al. (1992). It has two prominent, point-symmetric "arms" formed by pairs of condensations in addition to a bright internal elliptical structure. Some previous studies on NGC 6309 have determined an expansion velocity $V_{\exp }[\mathrm{OIII}]=34 \mathrm{~km} \mathrm{~s}^{-1}$ (Sabbadin 1984), as well as mean physical parameters and total abundances (Górny et al. 2004), namely, electron density and temperature $N_{\mathrm{e}}[\mathrm{S} \mathrm{II}]=2600 \mathrm{~cm}^{-3}$, $T_{\mathrm{e}}[\mathrm{N} \mathrm{II}]=12097 \mathrm{~K}, T_{\mathrm{e}}[\mathrm{O} \mathrm{III}]=11845 \mathrm{~K}$; and abundance 


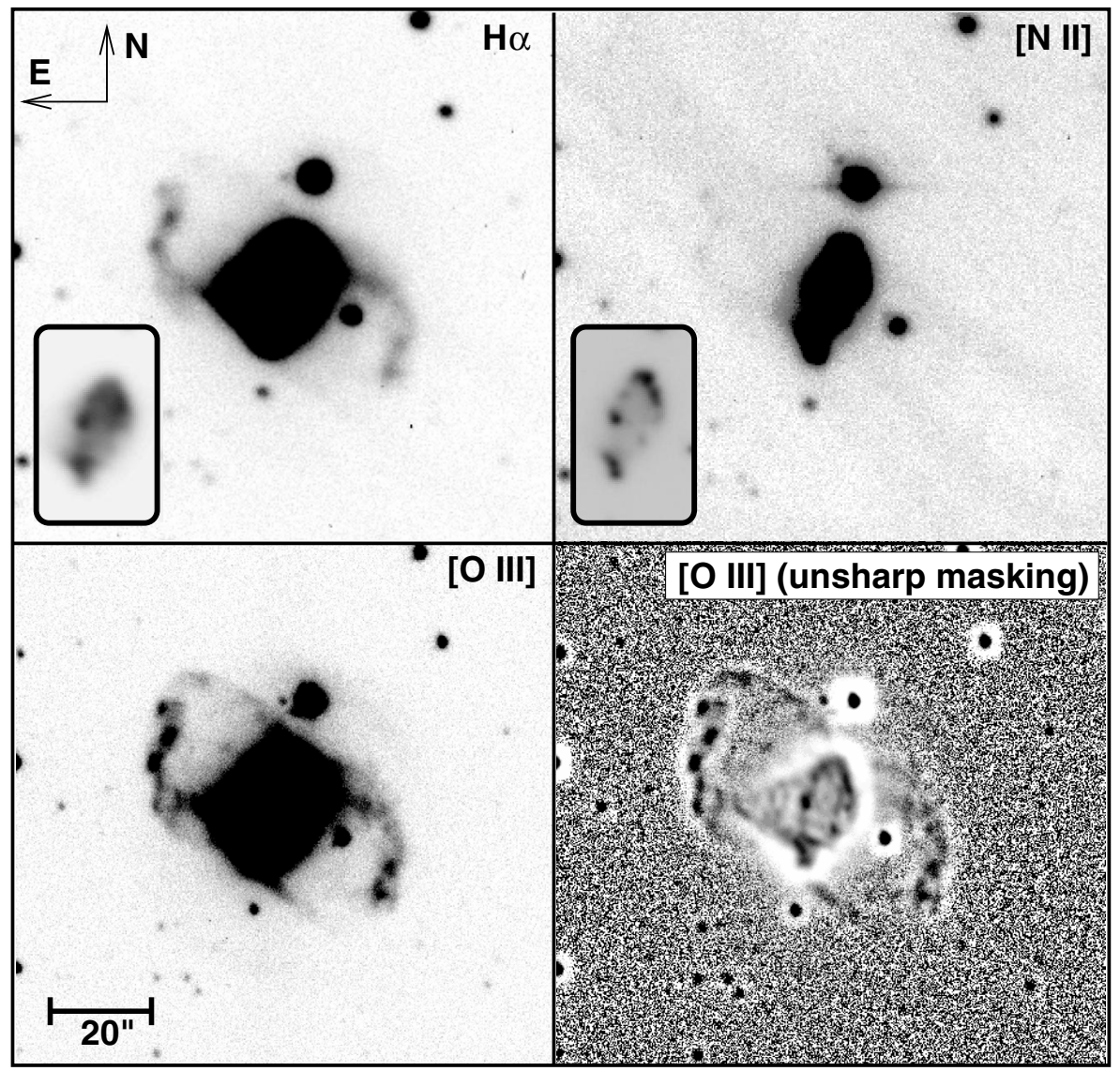

Fig. 1. Images of NGC 6309 in the light of $\mathrm{H} \alpha$ (top left), [N II] (top right) and [O III] (bottom left), including an unsharp masking of the [O III] image (bottom right). North is up and east is left in all the panels. Small frames are included inside the upper panels to show the structure of the central region.

ratios $\mathrm{He} / \mathrm{H}=0.1, \mathrm{~N} / \mathrm{H}=8.20, \mathrm{O} / \mathrm{H}=8.64, \mathrm{Ne} / \mathrm{H}=7.82$, and $\mathrm{S} / \mathrm{H}=6.49(\log \mathrm{H}=12$, except for $\mathrm{He} / \mathrm{H}$ ratio). Armour \& Kingsburgh (2001) found similar values. NGC 6309 has also been included in the list of PNe with low ionisation structures (LIS) by Gonçalves et al. (2003). Finally, the central star of NGC 6309 has been classified as a "weak emission line star" by Górny et al. (2004).

In spite of this suggestive morphology, an internal kinematic study of NGC 6309, as well as a full analysis of the physical conditions in the different nebular regions, has not yet been done. In this paper, we have carried out a radio-optical study of NGC 6309, including radio continuum mapping, optical imaging, and long-slit optical spectroscopy, in both, high- and lowdispersion. We discuss our results on the morphology, kinematics, physical conditions, ionic abundances, and nature of the gas emission to explore the possible mechanisms involved in the formation of NGC 6309.

\section{Observations and results}

\section{1. $C C D$ ground-based imaging}

We obtained narrow-band direct images with the Calar Alto Faint Object Spectrograph (CAFOS) in the $2.2 \mathrm{~m}$ telescope at Calar Alto Observatory $\left(\mathrm{CAHA}^{1}\right)$ in 1998 July 8 . We used two filters centered in $\mathrm{H} \alpha(\Delta \lambda=15 \AA)$ and $[\mathrm{N} \mathrm{II}] 6583(\Delta \lambda=20 \AA)$ as well as a CCD Loral with $2048 \times 2048$ pixels. The exposure time for both images was $1800 \mathrm{~s}$. The scale was 0.33 pixel $^{-1}$ and the seeing was about 1.'7. We obtained an additional image on

\footnotetext{
1 The Centro Astronómico Hispano-Alemán (CAHA) at Calar Alto, is operated jointly by the Max-Planck Institut für Astronomie and the Instituto de Astrofísica de Andalucía (CSIC).
}

2004 August 3 with the $1.5 \mathrm{~m}$ telescope at the Observatorio de Sierra Nevada ${ }^{2}$. The detector was a RoperScientific VersArray CCD with $2048 \times 2048$ pixels, each of 0 '.232 pixel $^{-1}$. We used an [O III]5007 ( $\Delta \lambda=50 \AA)$ filter with an exposure time of $900 \mathrm{~s}$. The images were reduced following standard procedures within the MIDAS package. In this case, seeing was 1.'3.

Figure 1 shows the images of $\mathrm{NGC} 6309$ in $\mathrm{H} \alpha$, [N II] and [O III]. An additional unsharp masking [O III] image is also presented in Fig. 1 to show the link between internal and external nebular structures. In these images, the high contrast between the brightness of the central region and that of the "arms" is evident. In particular, the lack of emission of [N II] from the "arms" is unexpectedly remarkable given that, in general, this kind of pointsymmetric microstructures is related to LIS (Gonçalves et al. 2003). However, there are also some cases in which the emission of [N II] is marginal (e.g. IC 5217; Miranda et al. 2006). In the case of NGC 6309, the lack of [N II] from the point-symmetric knots indicates that these cannot be considered as LIS.

The structure of the "arms" consists of at least four pairs of point-symmetric knots, some of them clearly extended perpendicular to their corresponding radial vector from the central star. The central region of NGC 6309 is a bright ellipse (major axis $\simeq 20^{\prime \prime}, \mathrm{PA}-14^{\circ}$ ) embedded in the main body of the nebula. The arms appear to leave from the vertexes of this ellipse. The unsharp masking [O III] image also shows a conelike structure with its base located on the central ellipse and the vertex on the NE arm. In [O III], faint emission connects the arms with the bright ellipse and seems to trace asymmetrical lobes. In

2 The Observatorio de Sierra Nevada is operated by the Consejo Superior de Investigaciones Científicas through the Instituto de Astrofísica de Andalucía (Granada, Spain). 


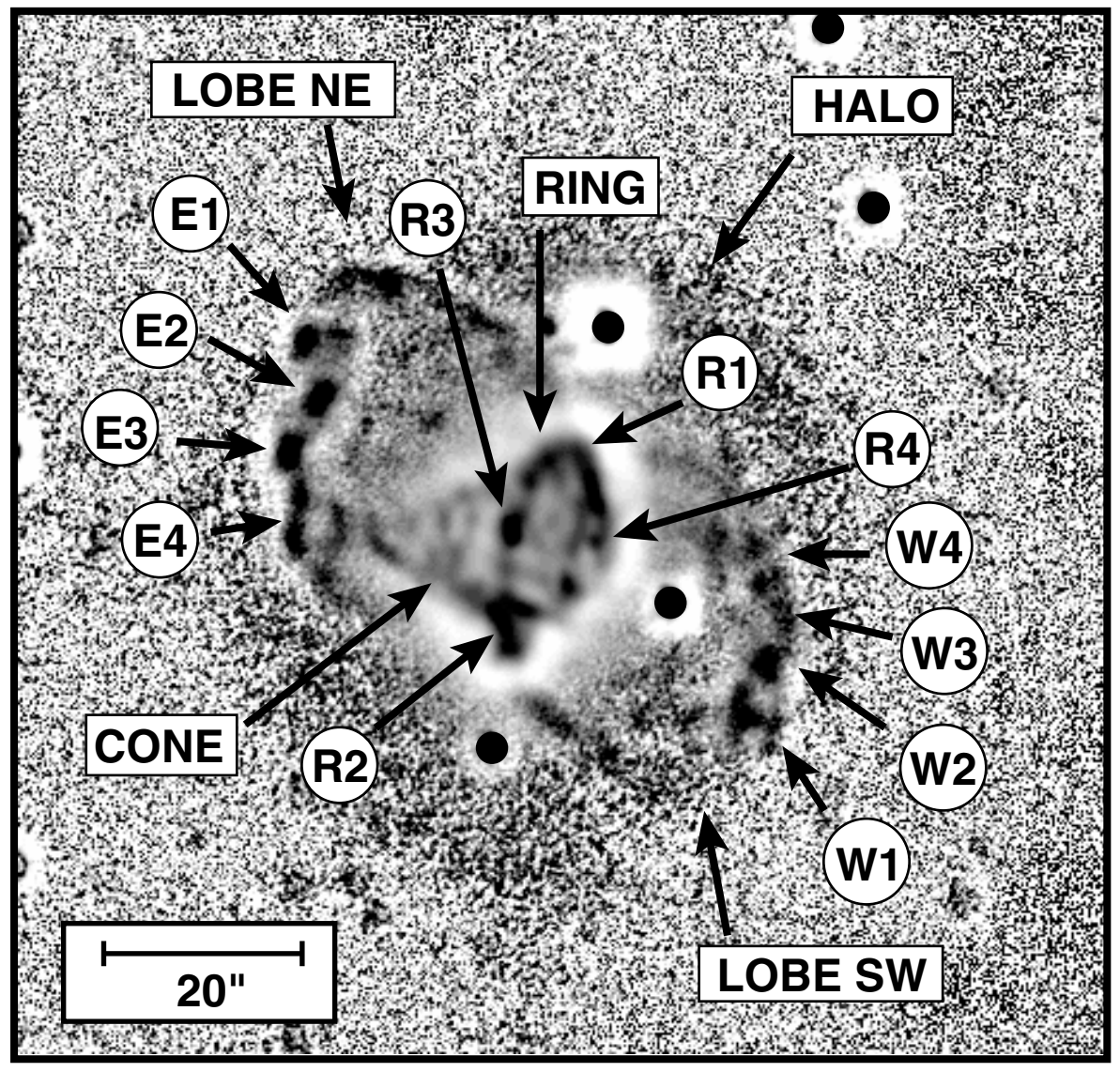

Fig. 2. Unsharp-masking image of NGC 6309 in the light of [O III]. North is up and east is left. Main morphological features as well as some regions of particular interest are pointed out and labeled.

fact, the appearance of NGC 6309 in [O III] resembles that of a quadrupolar $\mathrm{PN}$ in which one of the outflows (at $\mathrm{PA} 40^{\circ}$ ) protrude into the lobes of the other (at PA $76^{\circ}$ ). Part of the SW lobe appears to be open. Finally, a circular faint halo (probably spherical) is detected in [O III] image (Figs. 1 and 2). Its center coincides with the central star of the nebula and its size is $\simeq 56^{\prime \prime}$.

In Fig. 2, we show the unsharp masking [O III] image and label some morphological features that will be discussed later. Knots in the NE arm are named E1, E2, E3, and E4, whereas those in the SW arm are named W1, W2, W3, and W4. Regions in the ellipse are called $\mathbf{R} \mathbf{1}$ and $\mathbf{R} \mathbf{2}$ for the tips of its major axis, and $\mathbf{R 3}$ and $\mathbf{R 4}$ for those along the minor axis.

\subsection{HST-WFPC2 imaging}

An Hubble Space Telescope (HST) broadband image from the MAST Archive $^{3}$ was used in order to improve the general view of the internal morphology of NGC 6309 (proposal ID: 6119; PI: H. E. Bond; Date of observation: 1995 August 26). Figure 3 shows the $140 \mathrm{~s}$ image using the filter $F 555 W$ (nearly Johnson $V$ ). This image can be compared with the optical ground-based images in Fig. 1. The bright ellipse is formed by many clumps as well as diffuse gas. The ellipse is open in its SW and SE regions, whereas an apparently double structure is observed in the $\mathrm{S}$ and $\mathrm{N}$ regions. In addition, a system of faint

3 Some of the data presented in this paper were obtained from the Multimission Archive at the Space Telescope Science Institute (MAST). STScI is operated by the Association of Universities for Research in Astronomy, Inc., under NASA contract NAS5-26555. Support for MAST for non-HST data is provided by the NASA Office of Space Science via grant NAG5-7584 and by other grants and contracts.

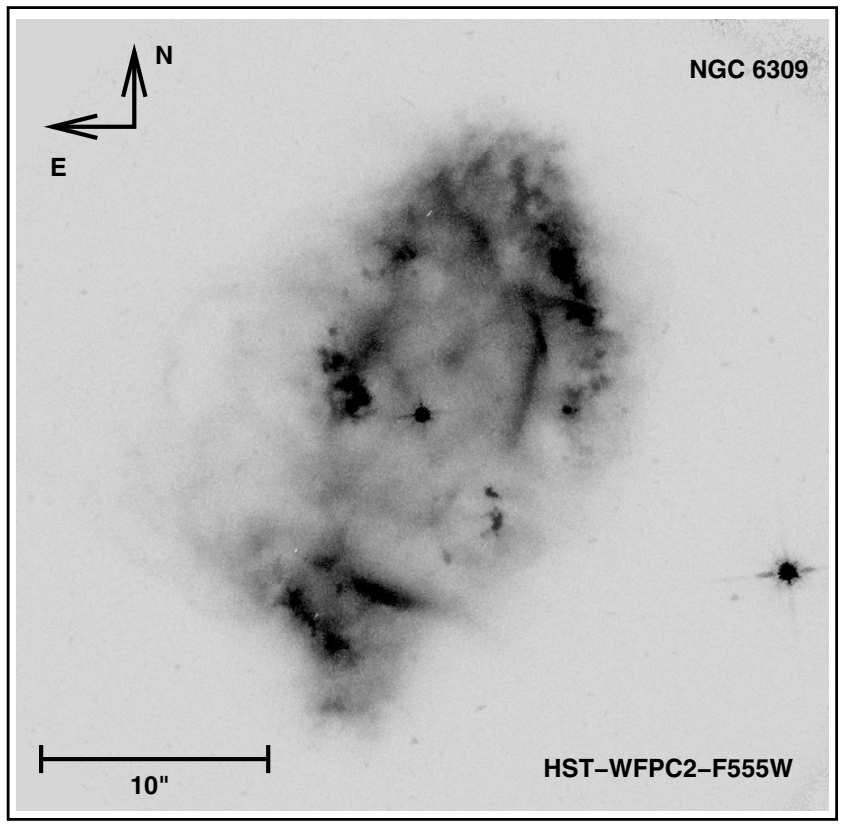

Fig. 3. HST broad-band CCD image of NGC 6309 in the filter F555W.

bubbles is detected towards the NE, which is probably related to the cone-like structure seen in [O III] (see above). Given the short exposure time of the HST image, evidence of the "arms" is very marginal. 


\section{3. $\lambda 3.6-\mathrm{cm} V L A-D$}

We obtained radio continuum observations at $\lambda 3.6-\mathrm{cm}$, toward NGC 6309 with the Very Large Array (VLA) of the National Radio Astronomy Observatory (NRAO) ${ }^{4}$ in the D configuration during 1996 August 8. The standard VLA continuum mode with a $100 \mathrm{MHz}$ bandwidth and two circular polarizations was employed. The flux and phase calibrators were $1331+305$ (adopted flux density 5.2 Jy) and 1733-130 (observed flux density $10.7 \mathrm{Jy})$, respectively. We set phase center at $\alpha(2000)=$ $17^{\mathrm{h}} 14^{\mathrm{m}} 03^{\mathrm{s}} .6, \delta(2000)=-12^{\circ} 54^{\prime} 37^{\prime \prime}$. The on-target integration time was $28 \mathrm{~min}$. We calibrated the data and processed it using standard procedures of the Astronomical Image Processing System (AIPS) package of the NRAO. We obtained a cleaned map of NGC 6309 using the task IMAGR of AIPS (parameter ROBUST $=-3$; Briggs 1995). Self-calibration was also performed resulting in a final synthesized beam of 13.'9 in diameter and rms noise of the map of $\sigma=33 \mu \mathrm{Jy}_{\text {beam }}{ }^{-1}$.

The detected radio continuum emission is related to the central region and appears partially resolved. Based on a 2-D Gaussian fit (task IMFIT in AIPS), we estimate a size of $\simeq 19^{\prime \prime} \times 16^{\prime \prime}$ at PA $=-27^{\circ}$ (see Fig. 4). The position of the intensity peak was found at $\alpha(2000)=17^{\mathrm{h}} 14^{\mathrm{m}} 04^{\mathrm{s}} .28$, $\delta(2000)=-12^{\circ} 54^{\prime} 37^{\prime \prime}$. The total flux density from the radio map is $\simeq 115-\mathrm{mJy}$, which is similar to those measured by Milne \& Aller (1982); Ratag \& Pottasch (1991); and Condon \& Kaplan (1998); at $2 \mathrm{~cm}$ (146-mJy); $6 \mathrm{~cm}$ (102-mJy); and $21 \mathrm{~cm}$ (132-mJy); respectively. We did not detect emission from the "arms".

We also derived the mean physical conditions, based on the formalism of Mezger \& Henderson (1967). Optically-thin emission and $T_{\mathrm{e}}=10000 \mathrm{~K}$ were assumed, obtaining the following results: $N_{\mathrm{e}}=1900 \mathrm{~cm}^{-3} ; M(\mathrm{H} \mathrm{II})=0.07 M_{\odot} ; E M=5.1 \times$ $10^{5} \mathrm{pc} \mathrm{cm}^{-6}$. We assumed distance to the nebula to be $2 \mathrm{kpc}$ as the average value from the different estimates, which range between 1.1 and $2.5 \mathrm{kpc}$ (e.g., Daub 1982; Phillips \& Pottasch 1984; Amnuel et al. 1984; Maciel 1984; Cahn et al. 1992).

\subsection{Long slit low-dispersion spectroscopy}

Low-dispersion optical spectra were obtained with the Boller \& Chivens spectrometer in the $2.1 \mathrm{~m}$ UNAM telescope at the San Pedro Mártir Observatory (OAN-UNAM) in 1999 July 19, 20 (grating of 300 lines/mm) and 2002 August 7, 8 (grating of 400 lines/mm). In all of these cases, a CCD Tek $1024 \times 1024$ was used as detector. We set the slit width to $220 \mu \mathrm{m}\left(1.6^{\prime \prime}\right)$. The spatial scale is $1 . .05$ pixel $^{-1}$ whereas the spectral scale is 3 and $4 \AA$ pixel $^{-1}$ for the gratings of 400 and 300 lines/mm, respectively. We set the slit at several position angles, which are labeled as C, J, K, L (grating of 300 lines/mm), and $\mathrm{A}, \mathrm{C}$, and $\mathrm{E}$ (grating of 400 lines/mm), in Fig. 5.

Table 1 shows the dereddened spectra (considering case $B$ of recombination) and physical conditions obtained with the FiveLevel Atom Diagnostics Package (nebular) from IRAF $^{5}$ (applying the extinction law by Howarth 1983 for dereddening) for the regions R1, R2, R3, R4, E2, W1, and W2.

${ }_{4}$ The National Radio Astronomy Observatory is a facility of the National Science Foundation operated under cooperative agreement by Associated Universities, Inc.

5 The Image Reduction and Analysis Facility (IRAF) is distributed by the National Optical Astronomy Observatories, which are operated by the Association of Universities for Research in Astronomy, Inc., under cooperative agreement with the National Science Foundation.

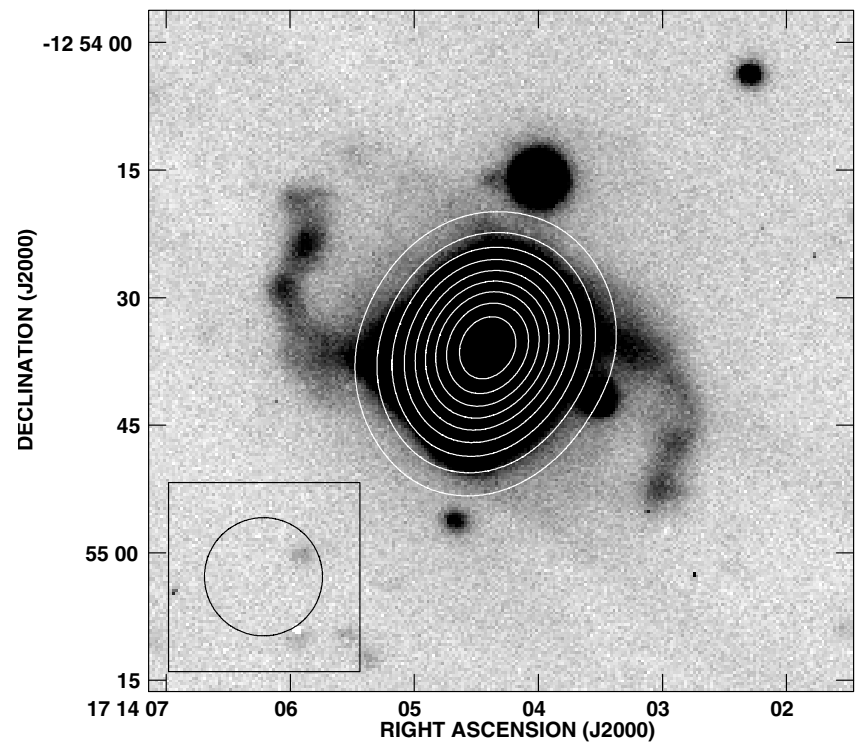

Fig. 4. Contour plot $\lambda 3.6-\mathrm{cm}$ continuum map of NGC 6309, overimposed on a $\mathrm{H} \alpha$ image. Contour levels are 10, 20, 30, 40, 50, 60, 70, 80 , and $90 \%$ of the peak flux $\left(6.3 \times 10^{-3} \mathrm{Jy}\right)$. The half-power beam width (13.'9 in diameter) is shown in the bottom-left corner.

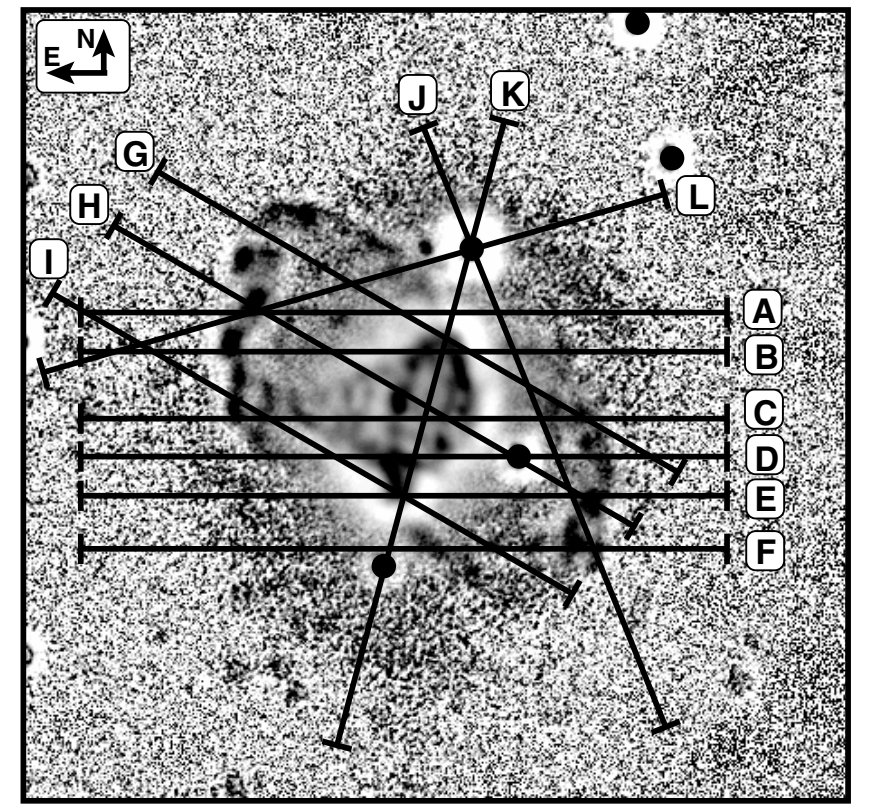

Fig. 5. Slits used in spectroscopy overimposed on [O III] unsharp masking image.

Physical conditions can only be determined for the regions of the central ellipse. Electron temperature and densities obtained from the high-excitation emission lines appears to be quite uniform $\left(T_{\mathrm{e}}[\mathrm{O} \mathrm{III}]=10750 \mathrm{~K}, N_{\mathrm{e}}[\mathrm{Cl} \mathrm{III}]=2350 \mathrm{~cm}^{-3}\right.$, $N_{\mathrm{e}}[\mathrm{Ar}$ IV $]=1430 \mathrm{~cm}^{-3}$, with variations within the estimated uncertainties. However, those physical parameters obtained from low-excitation emission ions appear as separated in two different regimes $\left(T_{\mathrm{e}}[\mathrm{N}\right.$ II $]=10200 \mathrm{~K}$, and $11800 \mathrm{~K} ; N_{\mathrm{e}}[\mathrm{S} \mathrm{III}]=$ $1620 \mathrm{~cm}^{-3}$ and $3800 \mathrm{~cm}^{-3}$ ).

The dereddened absolute $\mathrm{H} \beta$ flux values from regions R1, R2, R3, and R4 are similar (see Table 1). However, the corresponding logarithmic extinction coefficients $c_{\mathrm{H} \beta}$ spread from 0.70 to 0.97 (mean value including all regions is 0.87 ), probably due to differences in the internal dust distribution, as has been 
Table 1. Dereddened spectra and physical conditions from different regions in NGC 6309. Intensity values are relative to $I_{\mathrm{H} \beta}=100$. Extinction law $\left(f_{\lambda}\right)$ is from Howarth (1983). The size of the integration region (in arcsec) along the slit is 7'. 4 for $\mathbf{R 1}, \mathbf{R 2}, \mathbf{R 3}$, and $\mathbf{R 4}$, and $5^{\prime \prime} .3$ for the rest. Slit width was set at 1."6 in all the cases.

\begin{tabular}{|c|c|c|c|c|c|c|c|c|c|}
\hline Ion & $\lambda_{0}(\AA)$ & $f_{\lambda}$ & $\mathrm{R} 1$ & $\mathrm{R} 2$ & $\mathrm{R} 3$ & $\mathrm{R} 4$ & E2 & W1 & $\mathrm{W} 2$ \\
\hline$\overline{[\mathrm{O} \mathrm{II}]}$ & 3727 & 0.256 & 108 & 102 & - & - & - & - & - \\
\hline [Ne III] & 3869 & 0.230 & 163 & 165 & - & - & - & - & - \\
\hline [Ne III] & 3968 & 0.210 & 68 & 67 & - & - & - & - & - \\
\hline [S II $]$ & 4071 & 0.189 & 6.5 & - & - & - & - & - & - \\
\hline $\mathrm{H} \delta+\mathrm{He}$ II & 4101 & 0.182 & 33 & 34 & - & - & - & - & - \\
\hline $\mathrm{H} \gamma$ & 4340 & 0.127 & 55 & 53 & 26 & 27 & 52 & - & - \\
\hline [O III] & 4363 & 0.121 & 12 & 12 & 7.9 & 8.5 & - & - & - \\
\hline He I & 4471 & 0.095 & 8.2 & 7.1 & 3.4 & 3.2 & - & - & - \\
\hline He II & 4540 & 0.078 & - & - & 2.8 & 3.3 & - & - & - \\
\hline N III & 4640 & 0.054 & 4.3 & 5.0 & 5.2 & 6.1 & - & - & - \\
\hline He II & 4686 & 0.043 & 29 & 31 & 81 & 79 & 86 & 106 & - \\
\hline [Ar IV] & 4711 & 0.037 & 6.0 & 5.8 & 12 & 12 & - & - & - \\
\hline [Ar IV] & 4740 & 0.030 & 4.6 & 4.6 & 9.4 & 9.3 & - & - & - \\
\hline $\mathrm{H} \beta$ & 4861 & 0.000 & 100 & 100 & 100 & 100 & 100 & 100 & 100 \\
\hline [O III] & 4959 & -0.024 & 487 & 464 & 335 & 365 & 341 & 364 & 451 \\
\hline [O III] & 5007 & -0.036 & 1504 & 1466 & 993 & 1081 & 1046 & 1097 & 1238 \\
\hline N I & 5199 & -0.082 & 0.9 & - & - & - & - & - & - \\
\hline He II & 5411 & -0.133 & 2.5 & 2.3 & 6.2 & 6.0 & 8.5 & - & - \\
\hline [Cl III] & 5517 & -0.154 & 1.1 & 1.0 & 0.7 & 0.7 & - & - & - \\
\hline [Cl III] & 5537 & -0.157 & 1.0 & 1.0 & 0.7 & 0.6 & - & - & - \\
\hline [N II] & 5755 & -0.195 & 1.0 & 1.0 & 0.6 & - & - & - & - \\
\hline He I & 5876 & -0.215 & 13 & 12 & 7.1 & 8.1 & - & - & - \\
\hline [O I] & 6300 & -0.282 & $6.8^{a}$ & $7.2^{a}$ & 2.5 & 1.2 & - & - & - \\
\hline [S III] & 6312 & -0.283 & - & - & 1.8 & 1.7 & - & - & - \\
\hline [O I] & 6364 & -0.291 & 1.9 & 1.8 & 0.8 & 0.4 & - & - & - \\
\hline [Ar V] & 6435 & -0.302 & - & - & 1.4 & 1.4 & - & - & - \\
\hline [N II] & 6548 & -0.318 & 24 & 31 & 9.3 & 4.7 & - & - & - \\
\hline $\mathrm{H} \alpha$ & 6563 & -0.320 & 285 & 285 & 283 & 284 & 284 & 284 & 284 \\
\hline [N II $]$ & 6583 & -0.323 & 60 & 60 & 27 & 12 & 8.8 & - & - \\
\hline He I & 6678 & -0.336 & 3.4 & 3.6 & 2.4 & 2.6 & - & - & - \\
\hline [S II] & 6717 & -0.342 & 8.0 & 9.1 & 2.9 & 1.3 & - & - & - \\
\hline [S II] & 6731 & -0.344 & 11 & 12 & 4.8 & 2.1 & - & - & - \\
\hline [Ar V] & 7006 & -0.380 & - & - & 3.1 & 2.7 & - & - & - \\
\hline He I & 7065 & -0.387 & 3.1 & 3.1 & 1.9 & 2.0 & - & - & - \\
\hline [Ar III] & 7136 & -0.396 & 17 & 19 & 14 & 13 & 19 & 21 & 21 \\
\hline$[\mathrm{Ar} \mathrm{IV}]+[\mathrm{Fe} \mathrm{II}]$ & 7170 & -0.401 & 1 & - & 0.9 & 0.3 & - & - & - \\
\hline He II & 7178 & -0.401 & - & - & 0.9 & 0.7 & - & - & - \\
\hline [O II] & 7320 & -0.419 & $5.6^{b}$ & $5.7^{b}$ & 2.3 & 1.4 & - & - & - \\
\hline [O II $]$ & 7330 & -0.420 & - & - & 2.0 & 1.3 & - & - & - \\
\hline$\overline{c_{\mathrm{H} \beta}}$ & & & 0.96 & 0.97 & 0.70 & 0.80 & 0.86 & 0.85 & 0.97 \\
\hline $\log I_{\mathrm{H} \beta}$ & & & -13.4 & -13.5 & -13.2 & -13.4 & -15.2 & -15.3 & -15.9 \\
\hline$\left.\overline{T_{\mathrm{e}}[\mathrm{O}} \mathrm{III}\right]$ & $(\mathrm{K})$ & & 10800 & 10900 & 10700 & 10600 & - & - & - \\
\hline$T_{\mathrm{e}}[\mathrm{N}$ II $]$ & $(\mathrm{K})$ & & 10300 & 10100 & 11800 & - & - & - & - \\
\hline$N_{\mathrm{e}}[\mathrm{S}$ II $]$ & $\left(\mathrm{cm}^{-3}\right)$ & & 1800 & 1440 & 4000 & 3600 & - & - & - \\
\hline$N_{\mathrm{e}}[\mathrm{Cl}$ III $]$ & $\left(\mathrm{cm}^{-3}\right)$ & & 2100 & 2350 & 2600 & 1700 & - & - & - \\
\hline$N_{\mathrm{e}}[\mathrm{Ar} \mathrm{IV}]$ & $\left(\mathrm{cm}^{-3}\right)$ & & $>1000$ & $>1500$ & $>1700$ & $>1500$ & - & - & - \\
\hline$\overline{\log H} \alpha /[\mathrm{N}$ II $]$ & & & 0.533 & 0.499 & 0.889 & 1.244 & - & - & - \\
\hline $\log \mathrm{H} \alpha /[\mathrm{S}$ II $]$ & & & 1.182 & 1.142 & 1.565 & 1.913 & - & - & - \\
\hline $\log [\mathrm{O}$ III $] 5007 / \mathrm{H} \alpha$ & & & 0.723 & 0.711 & 0.545 & 0.580 & - & - & - \\
\hline $\log \lambda 6717 / \lambda 6731$ & & & 0.748 & 0.792 & 0.603 & 0.623 & - & - & - \\
\hline
\end{tabular}

${ }^{a}$ This emission line is blended with [S III] $6312 \AA$.

${ }^{b}$ This emission line is blended with [O II] $7330 \AA$.

Mean uncertainties are $\Delta c_{\mathrm{H} \beta}= \pm 0.02, \Delta T_{\mathrm{e}}= \pm 500 \mathrm{~K}, \Delta N_{\mathrm{e}}= \pm 400 \mathrm{~cm}^{-3}$.

found in other PNe (e.g. Vázquez et al. 1999b; Lee \& Kwok 2005).

In addition, we present the more common ratios for plasma diagnostics at the bottom of Table 1, which show that these regions are emitting by photoionisation-recombination processes with minimal or nonexistent shock-cooling contributions. This could be expected in the surroundings of a hot luminous star, however there are several cases of PNe in which shock-cooling processes make an important contribution to the microstructures emission (see Gonçalves 2003, for a compilation). For comparison, previous spectroscopic studies of NGC 6309 by Górny et al. (2004) give values of $c(\mathrm{H} \beta) \simeq 0.88, T_{\mathrm{e}}([\mathrm{O} \mathrm{III}]) \simeq 11845 \mathrm{~K}$, $T_{\mathrm{e}}([\mathrm{N} \mathrm{II}]) \simeq 12097 \mathrm{~K}$, and $N_{\mathrm{e}}([\mathrm{S} \mathrm{II}]) \simeq 2600 \mathrm{~cm}^{-3}$.

We have also estimated ionic and elemental abundances for some regions using IRAF and the ICF (Ionisation Correction Factors) method (Kingsburgh \& Barlow 1994), although we are 
Table 2. Ionic abundances of NGC 6309. In the case of ions with more than one transition, a flux-weighted average was performed. Electron temperature and density values of $T_{\mathrm{e}}=10700 \mathrm{~K}$ and $N_{\mathrm{e}}=2400 \mathrm{~cm}^{-3}$ were used on the calculations.

\begin{tabular}{lrrrrrrrr}
\hline \hline Ion & & $\mathrm{R} 1$ & $\mathrm{R} 2$ & $\mathrm{R} 3$ & $\mathrm{R} 4$ & $\mathrm{E}^{a}$ & $\mathrm{~W}^{a}$ & $\mathrm{~W}^{a}$ \\
\hline $\mathrm{He}^{+2}$ & & 0.02 & 0.03 & 0.07 & 0.06 & 0.06 & 0.09 & - \\
$\mathrm{He}^{+}$ & & 0.09 & 0.09 & 0.05 & 0.06 & - & - & - \\
\hline $\mathrm{O}^{+2}$ & $\left(\times 10^{-4}\right)$ & 4.68 & 4.93 & 2.02 & 3.78 & 3.66 & 3.82 & 4.41 \\
$\mathrm{O}^{+}$ & $\left(\times 10^{-5}\right)$ & 4.70 & 5.93 & 1.28 & 2.16 & - & - & - \\
$\mathrm{O}^{0}$ & $\left(\times 10^{-5}\right)$ & 1.13 & 1.29 & 0.27 & 0.25 & - & - & - \\
\hline $\mathrm{Ar}^{+4}$ & $\left(\times 10^{-7}\right)$ & - & - & 4.26 & 5.83 & - & - & - \\
$\mathrm{Ar}^{+3}$ & $\left(\times 10^{-7}\right)$ & 8.78 & 9.22 & 12.2 & 19.4 & - & - & - \\
$\mathrm{Ar}^{+2}$ & $\left(\times 10^{-6}\right)$ & 1.48 & 1.68 & 0.90 & 1.18 & 1.71 & 1.91 & 1.92 \\
\hline $\mathrm{N}^{+}$ & $\left(\times 10^{-5}\right)$ & 1.15 & 1.37 & 0.37 & 0.25 & 0.18 & - & - \\
$\mathrm{N}^{0}$ & $\left(\times 10^{-6}\right)$ & 2.18 & - & - & - & - & - & - \\
\hline $\mathrm{S}^{+2}$ & $\left(\times 10^{-6}\right)$ & - & - & 2.14 & 3.99 & - & - & - \\
$\mathrm{S}^{+}$ & $\left(\times 10^{-7}\right)$ & 5.29 & 5.87 & 2.07 & 1.31 & - & - & - \\
\hline $\mathrm{Ne}^{+2}$ & $\left(\times 10^{-4}\right)$ & 1.59 & 1.75 & - & - & - & - & - \\
$\mathrm{Cl}^{+2}$ & $\left(\times 10^{-7}\right)$ & 1.28 & 1.28 & 0.63 & 0.92 & - & - & - \\
\hline & & & & & & & &
\end{tabular}

aware that recently, Gonçalves et al. (2006) have noticed that elemental abundances derived by using this method are overestimated if they are obtained from narrow, long-slit spectra. The results are shown in Tables 2 and 3.

Regions $\mathbf{R} \mathbf{1}$ and $\mathbf{R} \mathbf{2}$ are similar in physical conditions as well as in ionic abundances. In both regions, the abundances of the three species of oxygen as well as $\mathrm{He}^{+}, \mathrm{Ar}^{+2}, \mathrm{~N}^{+}, \mathrm{S}^{+}$, and $\mathrm{Cl}^{+2}$ are enhanced with respect to the other regions. The opposite occurs for $\mathrm{Ar}^{+3}$ and $\mathrm{He}^{+2}$.

With reference to elemental abundances, these are consistent with the mean values for PNe and type I PNe, within uncertainties (see Table 3). The exception is N, which shows a deficiency, more in agreement with less-evolved objects, but consistent with previous estimates (Armour \& Kingsburgh 2001). Given the values of these abundances, NGC 6309 would be a type II PN (intermediate population), according to the classification of Peimbert (1978). This is reinforced by the low N/O ratio, which is in agreement with the expected value for a low-mass central star (0.6 $M_{\odot}$, according to Kwok 2000). If this interpretation of the abundances is right, NGC 6309 would represent another case of a non-type I PN with bipolar outflows (see Vázquez et al. 1999a). This strengthens the idea pointed out by Vázquez et al. that some low-mass central stars may also develop bipolar morphologies, in contrast to the fact that bipolar PNe are usually associated with relatively massive central stars (Corradi \& Schwarz 1995).

\subsection{Long slit high-dispersion spectroscopy}

We obtained high-dispersion optical spectra with the Manchester Echelle Spectrometer (MES; Meaburn et al. 2003) in the $2.1 \mathrm{~m}$ telescope $(f / 7.5)$ at the San Pedro Mártir observatory (OANUNAM) during the period of 2001 May 22, 23. We used a CCD SITe with $1024 \times 1024$ pixels as detector, and set the slit width to $150-\mu \mathrm{m}\left(1.6^{\prime \prime}\right)$. A $2 \times 2$ binning was used, resulting in a spatial scale of 0.6 pixel $^{-1}$ and a spectral scale of $0.1 \AA$ pixel $^{-1}$. We centered the spectral range at the $\mathrm{H} \alpha$ emission line. The spectra were wavelength calibrated with a Th-Ar arc lamp to an accuracy of $\pm 2 \mathrm{~km} \mathrm{~s}^{-1}$. The achieved spectral resolution, as indicated by the FWHM of the comparison lines, was $12 \mathrm{~km} \mathrm{~s}^{-1}$. Several spectra were obtained with the slit oriented E-W (slits A to F in Fig. 5).
We obtained on 2004 July 29, 30, a second series of spectra, using the same telescope and instrument. In this case, the spectra were centered on the [O III] $\lambda 5007$ emission line (slits G, H, I, and $\mathrm{K}$ in Fig. 5), and the spectral scale was $0.08 \AA$ pixel $^{-1}$.

Position-velocity (PV) maps for the $\mathrm{H} \alpha$ and [O III] $\lambda 5007$ are shown in Fig. 6. We note that the He II $\lambda 6560$ line is also observed in the PV maps at the slits B, C, and D. In the PV maps (Fig. 6) relative position is measured with respect to the central star whereas radial velocity is measured relative to the systemic velocity, for which we deduce $V_{\mathrm{LSR}}=-32 \pm 2 \mathrm{~km} \mathrm{~s}^{-1}$, based on the expansion velocity at the position of the central star ([O III] spectra from slits $\mathrm{H}$ and $\mathrm{K}$ ). This value is close to that reported by Schneider et al. (1983) of $V_{\mathrm{LSR}} \simeq-33.4 \pm 2.8 \mathrm{~km} \mathrm{~s}^{-1}$.

In a first step to analyse the high-resolution spectra, we extract from the PV maps the spatial position and radial velocity of the individual regions labeled in Fig. 2. The results are listed in Table 4. The knots in the arms present a noticeable pointsymmetry, both in their position with respect to the central star and in their radial velocity. In the case of the regions in the bright ellipse, $\mathbf{R} \mathbf{1}$ and $\mathbf{R} \mathbf{2}$ are moving at the systemic velocity while $\mathbf{R} 3$ and $\mathbf{R} 4$, located along the minor axis, present the maximum radial velocity in this structure.

In a second step, we analyse the emission-line features in the PV maps as a whole. Slits $\mathrm{H}$ and $\mathrm{K}$ are tracing the minor and major axes of the internal ellipse, respectively. In the PV map of slit H (Fig. 6), the regions E2 and W2 can be noted as bright condensations located at the extremes of the emissionline features. Maximum expansion velocity at the lobes can be measured as $2 v_{\text {exp }} \simeq 80 \mathrm{~km} \mathrm{~s}^{-1}$ (SW lobe expansion is best viewed in panel I). The lobe NE shows a bulk radial velocity of $\simeq+20 \mathrm{~km} \mathrm{~s}^{-1}$ (as measured at $20^{\prime \prime}$ from the center, panel $\mathrm{H}$ ), whereas the lobe SW presents $\simeq-20 \mathrm{~km} \mathrm{~s}^{-1}$ (as measured at $20^{\prime \prime}$ from the center, panel I). The central region also seems to be expanding, showing a maximum velocity splitting of $2 v_{\text {exp }} \simeq 69 \pm$ $2 \mathrm{~km} \mathrm{~s}^{-1}$, making difficult to determine the radial velocity of the microstructures R3 and R4 accurately. However, the PV map from slit $\mathrm{H}$ ([O III]) shows evidence of two additional components separated $\simeq 8^{\prime \prime}$ and $\simeq 46 \mathrm{~km} \mathrm{~s}^{-1}$ that could be these features (locations are indicated in Fig. 6 by a pair of arrows).

Slit K crosses the major axis of the ellipse. In the corresponding PV map, a velocity ellipse is observed. Features R1 and R2 move with the systemic velocity. However, small regions beyond the ellipse are also found with radial velocities of $\simeq \pm 30 \mathrm{~km} \mathrm{~s}^{-1}$. Maximum expansion velocity is observed at the center of the ellipse $\left(2 v_{\exp } \simeq 66 \pm 2 \mathrm{~km} \mathrm{~s}^{-1}\right)$, which is nearly compatible with previous spectroscopic studies (Sabbadin 1984, $\simeq 68 \pm 3 \mathrm{~km} \mathrm{~s}^{-1}$; Armour \& Kingsburgh 2001, $\simeq 64 \pm 5 \mathrm{~km} \mathrm{~s}^{-1}$ ).

From these slits ( $\mathrm{H}$ and $\mathrm{K}$ ), the presence of two expanding asymmetrical lobes and a velocity ellipse in the center is evident. The expansion of these bipolar lobes is confirmed with data from the other slits, and the kinematics of their borders is traced by that of the point-symmetric knots.

In the PV map from slit A, we detect an intense emission centered at $2^{\prime \prime}$ toward the west and relative velocity centered at $V_{\mathrm{r}} \simeq+20 \mathrm{~km} \mathrm{~s}^{-1}$. This feature corresponds to the upper border of the ellipse $\left(\simeq 5^{\prime \prime}\right.$ above R1). Faint emission from the halo is detected to the west of this feature (up to $\simeq 10^{\prime \prime}-25^{\prime \prime}$ ) in agreement with the observed size of this structure.

On the other hand, the PV map of slit I shows the radial velocity of feature $\mathbf{E} 4$ (the brightest feature) as $\simeq+24 \mathrm{~km} \mathrm{~s}^{-1}$, in addition to the expansion velocity of lobe $S W$ close to $\simeq 40 \mathrm{~km} \mathrm{~s}^{-1}$. Finally, the PV map of slit $\mathrm{C}$ covers the region at lobe NE corresponding to the cone-like structure. This region expands to 
Table 3. Elemental abundances of NGC 6309. ICFs were obtained following Kingsburgh \& Barlow (1994). Comparison with mean values of All-type PNe, type I PNe and other objects are shown. Except for He, all the abundances relative to $\mathrm{H}$ are logarithmic values with $H=+12$.

\begin{tabular}{|c|c|c|c|c|c|c|c|c|c|}
\hline Ion & R1 & $\mathrm{R} 2$ & R3 & R4 & $\mathrm{G}_{0} 4^{a}$ & $\mathrm{PNe}^{b}$ & $\mathrm{TIPNe}^{c}$ & $\overline{\mathrm{H} \text { II }}{ }^{d}$ & $\operatorname{Sun}^{e}$ \\
\hline$\overline{\mathrm{He} / \mathrm{H}}$ & $0.11 \pm 0.01$ & $.11 \pm 0.01$ & $0.12 \pm 0.01$ & $.12 \pm 0.01$ & $.10 \pm 0.01$ & $0.12 \pm 0.02$ & $0.13 \pm 0.04$ & $0.10 \pm 0.01$ & $0.09 \pm 0.01$ \\
\hline $\mathrm{O} / \mathrm{H}$ & $8.74 \pm 0.02$ & $.72 \pm 0.02$ & $8.72 \pm 0.01$ & $4 \pm 0.01$ & $8.64 \pm 0.02$ & $8.68 \pm 0$ & $65 \pm$ & $0 \pm$ & .05 \\
\hline $\mathrm{N} / \mathrm{H}$ & $7.93 \pm 0.02$ & $7.92 \pm 0.02$ & $8.04 \pm 0.01$ & $7.89 \pm 0.02$ & $8.20 \pm 0.12$ & $5 \pm 0$ & $2=$ & $7 \pm 0$ & \pm 0.06 \\
\hline $\mathrm{Ne} / \mathrm{H}$ & $8.26 \pm 0.02$ & $.25 \pm 0.02$ & - & - & $7.82 \pm 1.00$ & $3.09 \pm 0$ & $.09 \pm$ & $7.90 \pm$ & 0.06 \\
\hline $\mathrm{Ar} / \mathrm{H}$ & $6.40 \pm 0.10$ & $42 \pm 0.10$ & $.54 \pm 0.01$ & $.51 \pm 0.01$ & - & $6.39 \pm 0$ & $42 \pm$ & $6.42 \pm 0$ & \pm 0.08 \\
\hline $\mathrm{S} / \mathrm{H}$ & $6.74 \pm 0.01$ & $6.73 \pm 0.01$ & $6.84 \pm 0.01$ & $6.87 \pm 0.01$ & $6.49 \pm 0.05$ & $6.92 \pm 0.30$ & $6.91 \pm 0.30$ & $7.06 \pm 0.06$ & $7.14 \pm 0.05$ \\
\hline
\end{tabular}

${ }^{a}$ Taken from Górny et al. (2004).

${ }^{b}$ Average for PNe (Kingsburgh \& Barlow 1994).

${ }^{c}$ Average for type I PNe (Kingsburgh \& Barlow 1994).

${ }^{d}$ Average for H II regions (Shaver et al. 1983).

${ }^{e}$ The Sun (Grevesse et al. 2007).

Table 4. Basic kinematical data for the main morphological features in NGC 6309. Angular distance and position angle (PA) are measured from the central star. Radial velocity is measured with respect to the systemic velocity $\left(-32 \mathrm{~km} \mathrm{~s}^{-1}\right)$ from the emission line indicated in the last column. Uncertainties for the first row apply to the rest.

\begin{tabular}{|c|c|c|c|c|}
\hline Feature & $\begin{array}{l}\text { PA } \\
\left({ }^{\circ}\right)\end{array}$ & $\begin{array}{l}\text { Angular } \\
\text { distance } \\
\left({ }^{\prime \prime}\right)\end{array}$ & $\begin{array}{l}\text { Radial } \\
\text { velocity } \\
\left(\mathrm{km} \mathrm{s}^{-1}\right) \\
\end{array}$ & $\begin{array}{l}\text { Emission } \\
\text { line }\end{array}$ \\
\hline R1 & $-15 \pm 4^{\circ}$ & $9 \pm 3^{\prime \prime}$ & $0 \pm 2 \mathrm{~km} \mathrm{~s}^{-1}$ & [O III] \\
\hline R2 & +165 & 10 & 0 & [O III] \\
\hline R3 & +75 & 4 & +23 & [O III] \\
\hline R4 & -105 & 4 & -23 & [O III $]$ \\
\hline E1 & +50 & 30 & +35 & [O III] \\
\hline E2 & +60 & 26 & +36 & $\mathrm{H} \alpha,[\mathrm{O} \mathrm{III}]$ \\
\hline E3 & +70 & 27 & +35 & $\mathrm{H} \alpha$ \\
\hline E4 & +90 & 25 & +24 & $\mathrm{H} \alpha$ \\
\hline W1 & -130 & 27 & -34 & $\mathrm{H} \alpha$ \\
\hline W2 & -120 & 25 & -36 & $\mathrm{H} \alpha,[\mathrm{O} \mathrm{III}]$ \\
\hline W3 & -110 & 24 & -34 & $\mathrm{H} \alpha,[\mathrm{O} \mathrm{III}]$ \\
\hline W4 & -90 & 21 & -20 & $\mathrm{H} \alpha$ \\
\hline
\end{tabular}

$\simeq 25 \mathrm{~km} \mathrm{~s}^{-1}$, and is located from $5^{\prime \prime}$ to $15^{\prime \prime}$ toward the East from the center. The kinematics of the cone is practically indistinguishable from that of lobe NE.

\section{Discussion}

\subsection{The physical structure of NGC 6309}

The most reasonable explanation for the morphology and kinematics of the central ellipse is that such structure corresponds to a tilted expanding torus. Although other possible structures are possible, this interpretation appears to be a better fit to our data. Assuming that the torus is circular and its diameter is $\simeq 20^{\prime \prime}$, we deduce an inclination angle of the torus axis with respect to the line of sight of $66^{\circ}$. The expansion velocity of the torus, corrected by the inclination angle is $V_{\exp } \simeq 25 \mathrm{~km} \mathrm{~s}^{-1}$. Therefore, the size of this structure and its kinematic age are $\simeq 0.2 \mathrm{pc}$ and $3800 \mathrm{yr}$ respectively, assuming a distance of $2 \mathrm{kpc}$. In addition, the northeastern half of the torus is blueshifted whereas the southwestern half is redshifted. We note that these calculations have been obtained from the earth-based observations. Since the HST image shows a more complex structure in the torus, these results should be considered as an approximation only.

With regard to the bipolar lobes, it is clear that a model of a single bipolar system with a main axis cannot reproduce the observed morphology, given the apparent two directions observed in the nebula. Therefore, we have considered a quadrupolar model consisting of two pairs of bipolar lobes, one of them oriented at $\mathrm{PA}+76^{\circ}$, coincident with the torus axis, and the other at $\mathrm{PA}+40^{\circ}$, coinciding with the apparent protrusions in the lobes along this axis. Each of these bipolar systems is described following the formulation by Solf \& Ulrich (1985),

$V(\phi)=V_{\mathrm{e}}+\left(V_{\mathrm{p}}-V_{\mathrm{e}}\right) \times \sin ^{\alpha}(|\phi|)$,

where $\phi$ is the latitude angle above the equator; $V_{\mathrm{e}}$ and $V_{\mathrm{p}}$ are the equatorial and polar expansion velocity respectively; and $\alpha$ is an exponent that fits the specific hour-glass shape. With the main assumption that the axis of the torus and that of the bipolar ejection at $\mathrm{PA}+76^{\circ}$ are the same, the kinematics and morphology of this outflow can be reasonably reproduced with $V_{\mathrm{e}}=25 \mathrm{~km} \mathrm{~s}^{-1}$ (expansion velocity of the torus), $V_{\mathrm{p}}=75 \mathrm{~km} \mathrm{~s}^{-1}$, and $\alpha=6$. There is no clue about the inclination angle of the outflow at $\mathrm{PA}+40^{\circ}$. If we assume the same inclination angle, we find reasonable fits for this bipolar system with $V_{\mathrm{e}}=29 \mathrm{~km} \mathrm{~s}^{-1}, V_{\mathrm{p}}=86 \mathrm{~km} \mathrm{~s}^{-1}$, and $\alpha=6$. In Fig. 7, we show the hour-glass shapes deduced from these fits overimposed on the unsharp-masking image of NGC 6309. The observed shape, as well as the kinematics of the knots, are reasonably reproduced by the models. With the model data and the assumption of a distance of $2 \mathrm{kpc}$, we find a kinematical age of $4000 \mathrm{yr}$ for the bipolar lobes at PA $+40^{\circ}$ and of $3700 \mathrm{yr}$ for the bipolar system at PA $+76^{\circ}$. These ages are not very different from each other and are also similar to the age deduced for the torus. This suggests that the formation of the different structures in NGC 6309 has occurred in a relatively small time span. In addition, as these kind of outflows have been observed in proto-planetary nebulae (PPN, e.g. Sahai 1998), our results impose a minimal value of time $(\simeq 4000 \mathrm{yr})$ for the PPN to PN stage in this nebula. Other structures such as the "cone" and the halo, are present in this nebula, but we must conduct further studies to understand their physical properties.

\subsection{The formation of NGC 6309}

The appearance of NGC 6309, as well as the position of the point-symmetric knots perpendicular to a radial position vector from the central star, strongly resemble the point-symmetric structures observed in Fleming 1 (López et al. 1993). Cliffe et al. (1995) studied the interaction of a precessing jet with the interstellar medium. They find that point-symmetric structures can be formed by this interaction, and with the time, the individual bow-shocks can merge into a single shock structure. The ulterior evolution of such structures will lead to the formation of bipolar lobes with non-uniform brightness distribution being 


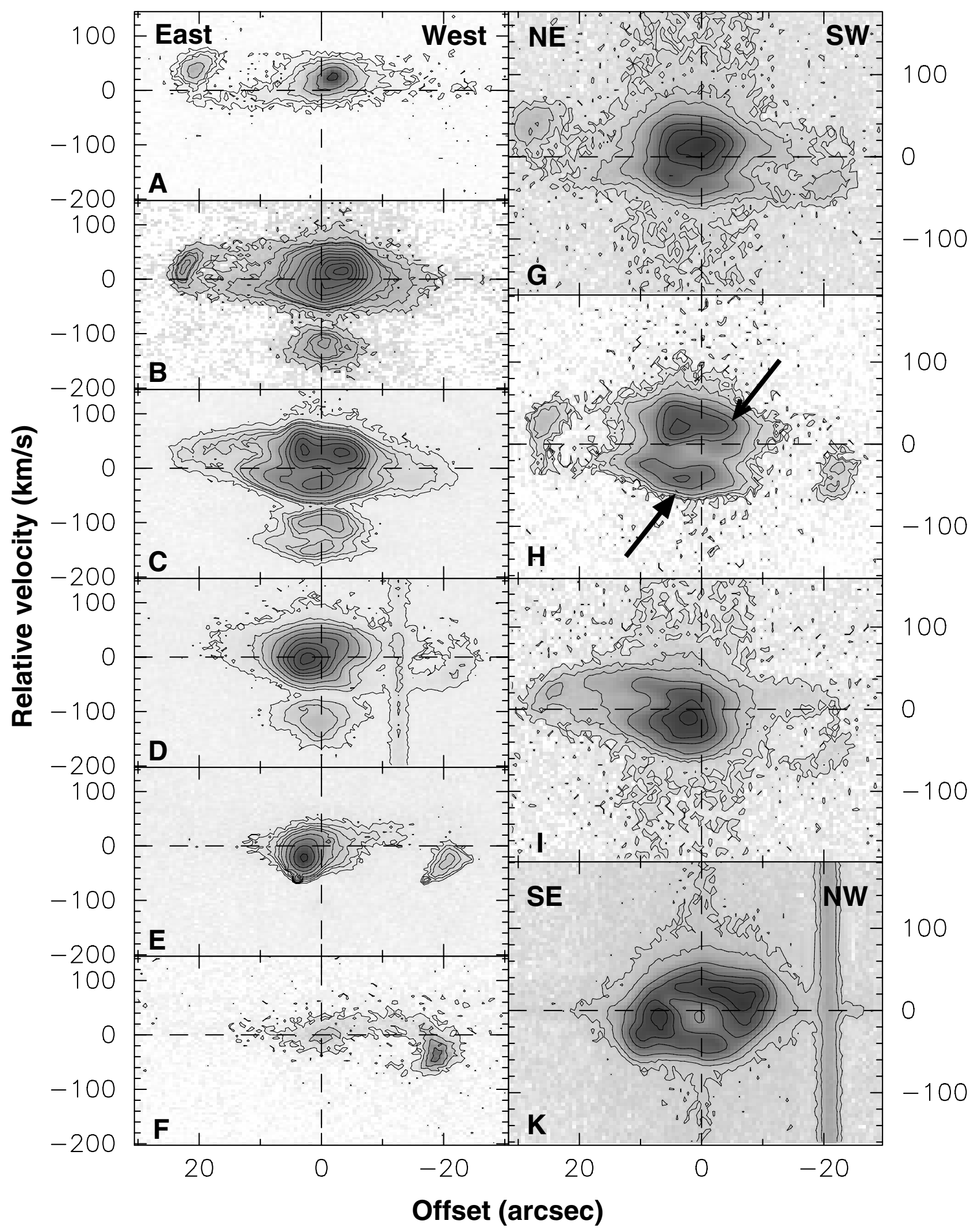

Fig. 6. Position-velocity gray/contour maps corresponding to the slits A to $\mathrm{F}$ in the light of $\mathrm{H} \alpha$ (left panels), and slits $\mathrm{G}, \mathrm{H}, \mathrm{I}$, and $\mathrm{K}$ in [O III] $\lambda 5007$ (right panels). Slit orientation is indicated, being the same for all the left panels. In the right panels orientation is indicated on panels for slits $\mathrm{G}$ (valid also for $\mathrm{H}$ and $\mathrm{I}$ ) and $\mathrm{K}$. Velocity scale is different in left and right panels. The vertical dashed lines represent the projected position of the central star perpendicular to the corresponding slit position. On each panel, the horizontal dashed line corresponds to the systemic radial velocity $\left(V_{\mathrm{LSR}}=-32 \pm 2 \mathrm{~km} \mathrm{~s}^{-1}\right)$. Arrows in panel $\mathrm{H}$ indicate location of two features explained in the text. 


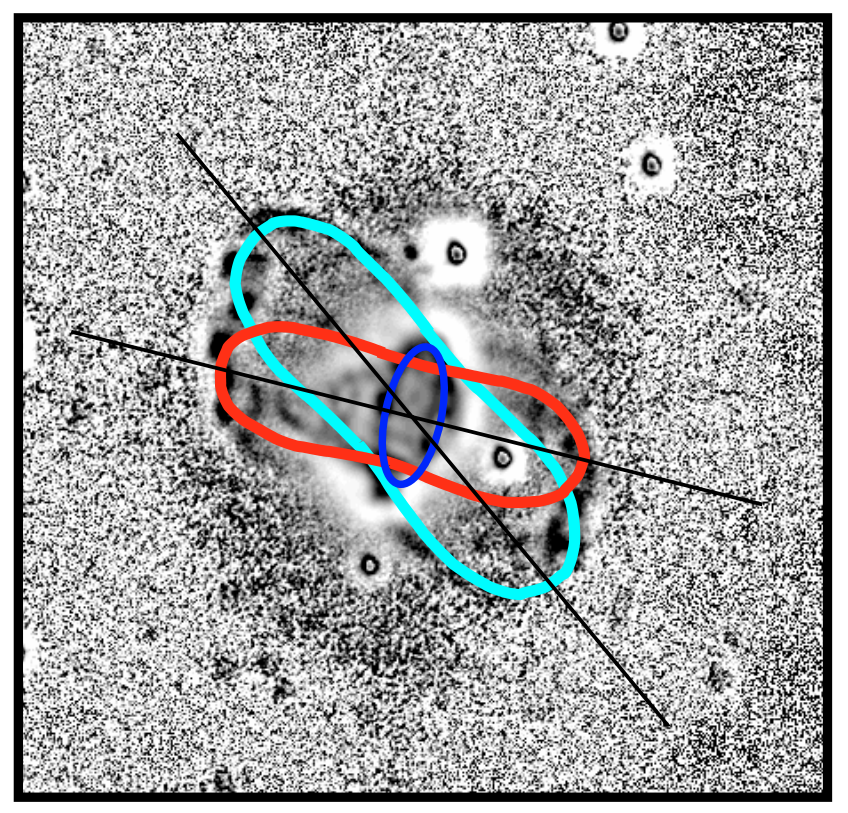

Fig. 7. NGC 6309 and the two bipolar outflows model. The fit of two hour-glass models is overimposed on the [O III] unsharp-masking image presented before. The central torus is also drawn.

point-symmetric respect to the central star. On the other hand, García-Segura \& López (2000) made models of bipolar PNe with point-symmetric structures using a steady misalignment of the magnetic collimation axis with respect to the symmetry axis of the bipolar outflow. In their models, such morphology is also produced by the action of jets.

A possible scenario for the formation of NGC 6309 is that the knots are the remnants of high-velocity bipolar collimated outflows that were ejected in the proto-planetary nebula (PPN) stage. There is evidence that precessing jets in young protoPN carve bipolar or multipolar cavities in the initial stage of PNe evolution (e.g. Sahai \& Trauger 1998; Sahai et al. 2005; Sánchez-Contreras et al. 2006). In such a scenario, the precessing jet is ejected during the proto-PN phase and after that, subsequently, it is stopped and cooled by its surroundings. The knots follow the thermal expansion of the bipolar lobes. In this case, the brightness of the knots comes mainly from the photoionisation process, as the shocked-cooled emission has turned off or it is hidden by the effect of a high-excitation photon source. This could explain the lack of [N II] emission in the point-symmetric knots.

In addition, we constructed a $[\mathrm{O} \mathrm{III}] / \mathrm{H} \alpha$ ratio image from images in Fig. 1. Such ratio image can be used as a diagnostic tool, as has been shown by Medina et al. (2007). Figure 8 shows the $[\mathrm{O} \mathrm{III}] / \mathrm{H} \alpha$ ratio image in which an enhancement of the [O III] emission is seen in the edge of the lobes, clearly related to the point-symmetric knots. It is noticeable that the emission vanished at the end of the SW lobe, possibly corresponding to a breaking of the shell due to the action of the outflow. According to Medina et al. (2007), the [O III] enhancement would be compatible with structures shaped by collimated outflows, as in the case of IC 4634. Finally, the circular halo probably corresponds to the remnant of the envelope ejected as a slow wind from the central star when it was at its AGB phase.

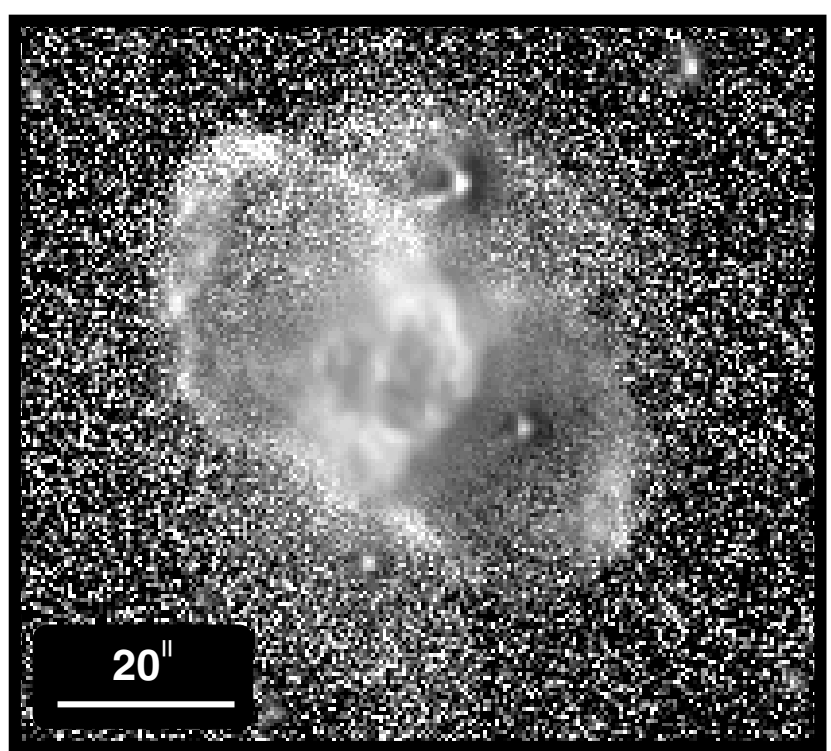

Fig. 8. $[\mathrm{O} \mathrm{III}] / \mathrm{H} \alpha$ ratio image. White represents high values of the ratio.

\section{Conclusions}

We have carried out an analysis of the planetary nebula NGC 6309, based on ground-based and space-based imaging, high- and low-dispersion spectroscopy, as well as VLA-D radio continuum. We summarize the main conclusions of this work as follows.

NGC 6309 can be described as a quadrupolar PN formed by a bright central torus, two systems of bipolar lobes oriented at different directions, and point-symmetric knots that trace the edges of the lobes. The torus expands at $25 \mathrm{~km} \mathrm{~s}^{-1}$, whereas the polar expansion velocity of the lobes is $75 \mathrm{~km} \mathrm{~s}^{-1}$ for a first bipolar system at PA $76^{\circ}$, and $86 \mathrm{~km} \mathrm{~s}^{-1}$ for another bipolar system at PA $40^{\circ}$. Assuming a distance of $2 \mathrm{kpc}$ for the nebula, the kinematic ages of the structures ranges from 3700 to $4000 \mathrm{yr}$, suggesting that they have been formed in a short time span. The knots at the edges of the lobes suggest that the lobes have been formed by rapidly precessing bipolar jets that carved cavities in the previous red giant envelope. In addition to these structures, we detect a circular halo surrounding the torus and bipolar lobes, which probably corresponds to the envelope ejected in the AGB phase by the central star. There is also a conelike structure embedded in one of the lobes and with its base on the torus.

We also study internal variations of the physical conditions and chemical abundances in NGC 6309. The low-dispersion spectra indicate a high-excitation nebula, with low to medium variations of its internal physical conditions $(10600 \mathrm{~K} \lesssim$ $T_{\mathrm{e}}[\mathrm{O}$ III $] \lesssim 10900 \mathrm{~K} ; 10100 \mathrm{~K} \lesssim T_{\mathrm{e}}[\mathrm{N} \mathrm{II}] \lesssim 11800 \mathrm{~K}$; $1440 \mathrm{~cm}^{-3} \lesssim N_{\mathrm{e}}[\mathrm{S}$ II $] \lesssim 4000 \mathrm{~cm}^{-3} ; 1700 \mathrm{~cm}^{-3} \lesssim N_{\mathrm{e}}[\mathrm{Cl}$ III $] \lesssim$ $2600 \mathrm{~cm}^{-3} ; 1000 \mathrm{~cm}^{-3} \lesssim N_{\mathrm{e}}$ [ArIV] $\lesssim 1700 \mathrm{~cm}^{-3}$ ). The radio continuum emission indicates a mean electron density of $\simeq 1900 \mathrm{~cm}^{-3}$; emission measure of $5.1 \times 10^{5} \mathrm{pc} \mathrm{cm}^{-6}$; and an ionised mass $M(\mathrm{HII}) \simeq 0.07 M_{\odot}$. The logarithmic extinction coefficient $c_{\mathrm{H} \beta}$ range from 0.70 to 0.97 (mean value 0.87 ), which probably is produced by differences in the internal dust distribution.

Acknowledgements. R.V., S.A., L.O., M.E.C., and P.F.G. were supported by grants 32214-E and 45848 (CONACYT), and by grants IN114199 and IN111903-3 (PAPIIT-DGAPA-UNAM). L.F.M. and S.A. were supported by grant AYA2005-01495 of the Spanish MEC (cofunded by FEDER funds) and by Junta de Andalucia grant FQM1747. J.M.T. acknowledges partial financial support from the Spanish grant AYA2005-08523-C03. We are grateful 
to the staff of all the astronomical facilities used in this research, namely: (a) Centro Astronómico Hispano-Alemán, (b) Very Large Array of National Radio Astronomy Observatory, (c) Hubble Space Telescope Data Archive, (d) Observatorio Astronómico Nacional, operated by Universidad Nacional Autónoma de México, and (e) Observatorio de Sierra Nevada (IAA-CSIC). We thank the anonymous referee for critically reading the manuscript and for useful suggestions. We also thank fruitful discussions with Dr. Martín A. Guerrero (IAA-CSIC) and Prof. Mauricio Tapia (IA-UNAM). This research has made use of the SIMBAD database, operated at CDS, Strasbourg, France.

\section{References}

Amnuel, P. R., Guseinov, O. H., Novruzova, H. I., \& Rustamov, Y. S. 1984 Ap\&SS, 107, 19

Armour, M. H., \& Kingsburgh, R. L. 2001, RevMexAASC, 10, 38

Balick, B. 1987, AJ, 94, 671

Briggs, D. S. 1995, Ph.D. Thesis, New Mexico Inst. of Mining and Technology

Cliffe, J. A., Frank, A., Livio, M., \& Jones, T. W. 1995, ApJ, 447, L49

Cahn, J. H., Kaler, J. B., \& Stanghellini, L. 1992, A\&AS, 94, 399

Condon, J. J., \& Kaplan, L. D. 1998, ApJS, 117, 361

Corradi, R. L. M., \& Schwarz, H. E. 1995, A\&A, 293, 871

Daub, C. T. 1982, ApJ, 260, 612

García-Segura, G., \& López, J. A. 2000, ApJ, 544, 336

Gonçalves, D. R., Corradi, R. L. M., Mampaso, A., \& Perinotto, A. 2003, ApJ, 597,975

Gonçalves, D. R., Ercolano, B., Carnero, A., Mampaso, A., \& Corradi, R. L. M. 2006, MNRAS, 365, 1039

Górny, S. K., Stasinska, G., Escudero, A. V., \& Costa, R. D. D. 2004, A\&A, 427, 231

Grevesse, N., Asplund, M., \& Sauval, A. J. 2007, Space Sci. Rev., 130, 105

Howarth, I. D. 1983, MNRAS, 203, 301

Icke, V. 1988, A\&A, 202, 177

Kahn, F. D., \& West, K. A. 1985, MNRAS, 2012, 837

Kingsburgh, R. L., \& Barlow, M. J. 1994, MNRAS, 295, 75

Kwok, S. 2000, The origin and evolution of planetary nebulae (Cambridge, New York: Cambridge University Press), Cambridge Astrophys. Ser., 33

Kwok, S., Purton, C. R., \& Fitzgerald, P. M. 1978, ApJ, 219, L125

Lee, T. -H., \& Kwok, S. 2005, ApJ, 632, 340

Livio, M., \& Pringle, J. E. 1996, ApJ, 465, L55

Livio, M., \& Pringle, J. E. 1997, ApJ, 486, 835

López, J. A., Meaburn, J., \& Palmer, J. W. 1993, ApJ, 415, L135
López, J. A., Vázquez, R., \& Rodríguez, L. F. 1995, ApJ, 455, L63

Maciel, W. J. 1984, A\&AS, 55, 253

Meaburn, J., López, J. A., Gutiérrez, L., et al. 2003, Rev. Mex. Astron. Astrofis., 39,185

Mellema, G. 1995, MNRAS, 277, 173

Medina, J. J., Guerrero, M. A., Luridiana, V., et al. 2007, in Asymmetrical Planetary Nebulae IV, ed. R. L. M. Corradi, A. Manchado, \& N. Soker, Proceedings in electronic book (reference will be published in ADS)

Mezger, P. G., \& Henderson, A. P. 1967, A\&A, 147, 471

Milne, D. K., \& Aller, L. H. 1982, A\&AS, 50, 209

Miranda, L. F., \& Solf, J. 1992 A\&A, 260, 397

Miranda, L. F., Ayala, S., Vázquez, R., \& Guillén, P. F. 2006 A\&A, 456, 591

Peimbert, M. 1978, in Planetary Nebulae, ed. Y. Terzian (Dordrecht: Reidel), IAU Symp., 76, 215

Perinotto, M., Schönberner, D., Steffen, M., \& Calonaci, C. 2004, A\&A, 414, 993

Phillips, J. P., \& Pottasch, S. R. 1984, A\&A, 130, 91

Ratag, M. A., \& Pottasch, S. R. 1991, A\&AS, 91, 481

Rijkhorst, E.-J., Icke, V., \& Mellema, G. 2004, in Asymmetrical Planetary Nebulae III, ed. M. Meixner, J. Kastner, N. Soker, \& B. Balick, ASP Conf. Ser., 313, 472

Rijkhorst, E.-J., Mellema, G., \& Icke, V. 2005, A\&AS, 444, 849

Sabbadin, F. 1984, A\&AS, 58, 273

Sahai, R., \& Trauger, J. T. 1998, AJ, 116, 1357

Sahai, R., Le Mignant, D., Sánchez-Contreras, C., Campbell, R. D., \& Chafee, F. H. 2005, ApJ, 622, L53

Sánchez-Contreras, C., Le Mignant, D., Sahai, R., Chaffee, F. H., \& Morris, M. 2006 in Planetary Nebulae in our Galaxy and Beyond, ed. M. J. Barlow, \& R. H. Mendez (Cambridge University Press), IAU Symp., 234, 71

Schneider, S. E., Terzian, Y., Purgathofer, A., \& Perinotto, M. 1983, ApJS, 52, 399

Schwarz, H. E., Corradi, R. L. M., \& Melnick, J. 1992, A\&AS, 96, 23

Shaver, P. A., McGee, R. X., Newton, L. M., Danks, A. C., \& Pottasch, S. R. 1983, MNRAS, 204, 53

Schönberner, D., Jacob, R., Steffen, M., et al. 2005, A\&A, 431, 963

Schönberner, D., Jacob, R., Steffen, M., \& Sandin, C. 2007, A\&A, 473, 467

Solf, J., \& Ulrich, H. 1985, A\&A, 148, 274

Stanghellini, L., Corradi, R. L. M., \& Schwartz, H. E. 1993, A\&A, 276, 463

Vázquez, R., López, J. A., Miranda, L. F., Torrelles, J. M., \& Meaburn, J. 1999a, MNRAS, 308, 939

Vázquez, R., Torrelles, J. M., Rodríguez, L. F., et al. 1999b, ApJ, 515, 633

Vázquez, R., Miranda, L. F., Torrelles, J. M., et al. 2002, ApJ, 576, 860 